\title{
Automating the Discovery of Stable Domain Abstractions for Reusable Aspects
}

\author{
Dimitri Van Landuyt, Eddy Truyen and Wouter Joosen \\ DistriNet, Katholieke Universiteit Leuven \\ Celestijnenlaan 200A \\ B-3001 Leuven, Belgium \\ \{dimitri.vanlanduyt,eddy.truyen,wouter.joosen\}@ cs.kuleuven.be
}

Domain-driven aspect design aims to support effective reuse of aspects within a product-line or family of software systems. In this paper, we explore the feasibility of automating our approach to discover stable domain abstractions in requirements for the purpose of defining reusable pointcuts. This approach was introduced in earlier work mainly as a manual method. This paper defines an algorithmic procedure for each activity, and discusses to which degree each activity can be automated. The generalization activity (activity 3 ) is given specific attention, as it is the most complex activity at the heart of the approach. The end result is an algorithmic procedure for (semi-)automatically discovering stable domain abstractions to design effectively reusable pointcuts.

\section{Introduction}

The key principle behind Domain-Driven Design [3] is that stable abstractions in the problem domain form an important means of communication between the different members of a development team, as this problem domain is often the only common ground between the different developers. Stable domain abstractions are defined as key concepts of the problem domain that change slowly [11], and thus can be considered to be a ubiquitous vocabulary that is shared between the different stakeholders and designers.

By letting aspect composition specifications depend on such stable domain abstractions, this Domain-Driven Design approach offers an interesting base for dealing with fragility issues in the context of AOSD [5]. Additionally, the use of stable domain abstractions has been linked to effective reuse in literature $[11,7,14]$; i.e. pointcuts exposing stable domain abstraction are more likely to be reusable, and therefore are important assets in the definition of aspectoriented product lines architecture [6].

However, an important problem in this space is the need for automated process support, which allows the exploration and identification of such stable abstractions from domain models in a systematic way.
In earlier work [14], we have introduced such a domaindriven aspect design approach mainly as a manual method. In this paper, we examine the feasibility of automating this method to discover stable domain abstractions from domain models and use cases. The approach consists of seven activities. For each of these activities, we provide an algorithmic procedure and discuss to which degree this procedure can be automated.

This paper is structured as follows: Section 2 shortly outlines the method. Section 3 provides an algorithmic procedure for each activity, and assesses to which degree the activity can be automated. Section 4 discusses related work. Finally, Section 5 concludes the paper.

\section{Overview of the Method}

The activity diagram of Figure 1 presents the method.

In the first activity, requirements are gathered and modeled as use cases. A domain model is defined in parallel, as the approach relies heavily on its existence.

In activity 2 , crosscutting concerns are identified in the use case models. After this, the use cases are re-engineered so that each use case is affected by at most one concern (separation of concerns). To achieve this, the use case $e x$ tension relationship is employed. For this, extension points are introduced in the base use cases.

This typically results in highly coupled use case models. The goal of activity 3 is to apply generalization in the use case models to manage this high coupling more efficiently. This results in multiple use case hierarchies of which the roots are abstract use cases with generalized extension points.

Activity 4 is the final requirements engineering activity in our approach. During this activity, the generalized extension points are used to define domain abstractions. This definition contains a name and references to other domain concepts that are required for the particular compositioncontext: the contextual information. 


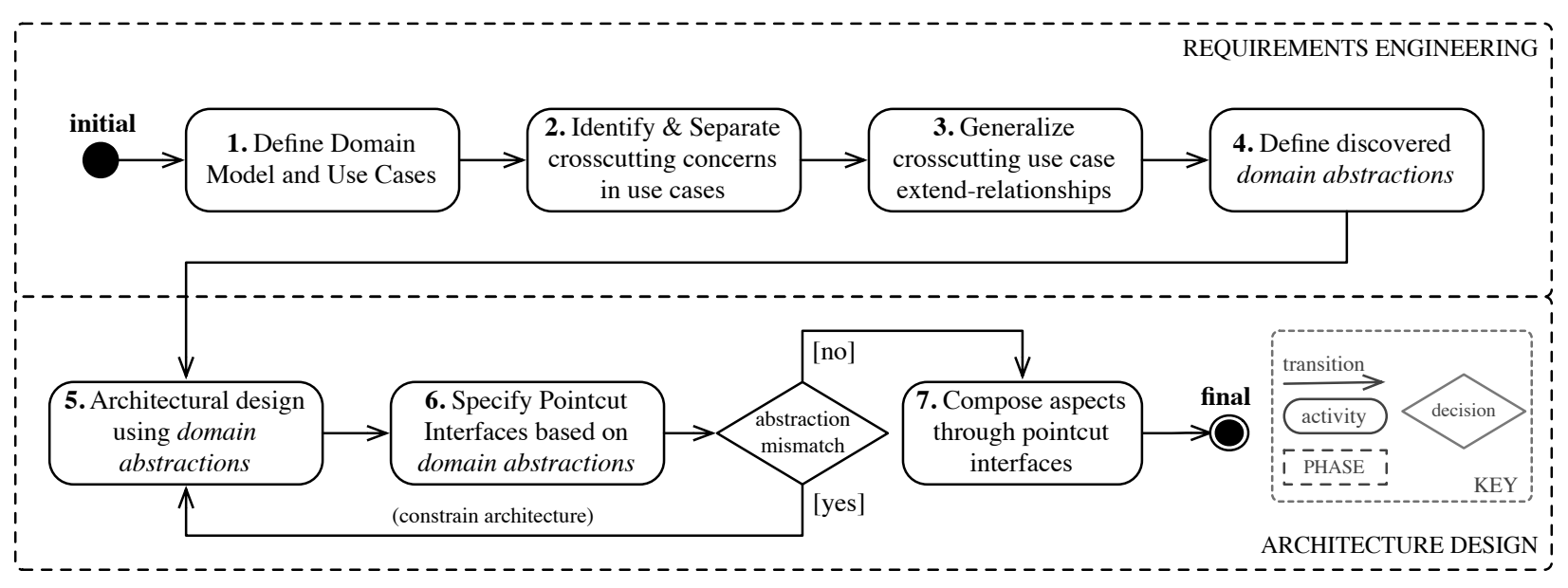

Figure 1: Overview of the method.

In activity 5 , the first architectural design activity, the architecture is created with the earlier-defined domain $a b$ stractions in mind: the architecture is constrained to ensure that it remains compatible with the domain abstractions.

Activity 6 involves mapping domain abstractions to pointcut interfaces. A pointcut interface has a scope that is limited to a module in the architecture (typically component, or a set thereof). In case a mismatch between an abstraction and the initial architecture prohibits the correct specification of the pointcut interface, the previous activity (activity 5) has to be repeated. Once the abstraction mismatch is corrected, the pointcut interface specification can be completed (repeat of activity 6 ).

In activity 7 , the final architecture design activity, aspectual compositions are specified to connect the advice to the pointcut interfaces.

For a more detailed description of the method, please refer to [14].

\section{Algorithmic procedure}

In this section, we zoom in on the requirements engineering activities (activities 1-4) of the method. As the algorithms presented in this Section rely heavily on use case terminology, we first present a metamodel for use cases. Then, for each of the activities we provide (i) the functions and procedures ${ }^{1}$ on which the algorithm relies, (ii) the algorithm itself, (ii) an illustration, and (iii) a discussion about the feasibility to automate it. Together, these provide an assessment of how feasible it is to automate the method as a whole.

\footnotetext{
${ }^{1}$ Distinction: a procedure changes the state of the use case diagrams, while a function calculates a result without changing state.
}

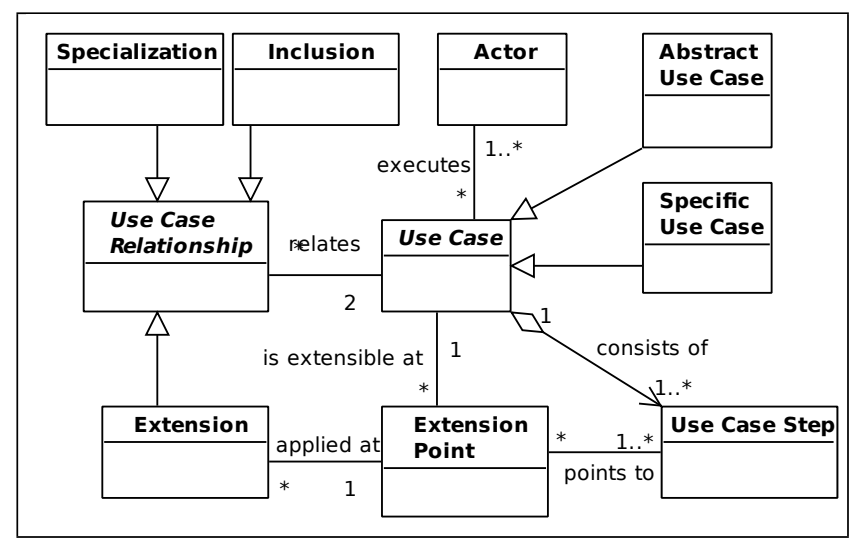

Figure 2: The metamodel for use cases (subset).

\section{Use Case Terminology and Metamodel}

The metamodel for use cases [4] is presented in Figure 2. Note that we only provide a subset of the full use case metamodel, as we focus on introducing the terms and concepts required to understand the algorithms.

A use case is a unit of functionality offered to the actor, which is the user interacting with the system. A use case consists of use case steps. It is possible to introduce an inheritance hierarchy by introducing abstract use cases and specific use cases that are related together by means of the specialization use case relationship. Another use case relationship is the use case extension. In this relationship, one use case is extended by another use case at a well-defined extension point which points to one or more use case steps. In this paper, we use the term 'abstract extension point' to denote an extension point in an abstract 
use case. Also, we use the term 'base use case' for a use case that is extended by another use case, which we call 'aspect use case'.

\section{Activity 1. Define Domain Model and Use Cases}

This activity is a requirements gathering and engineering process by nature. It involves a wide range of activities such as conducting stakeholder interviews, summarizing and generalizing requirements, resolving and prioritizing conflicting requirements, ....

The result of this activity is a decomposition of the problem space: both (i) functionally, in use cases and their interrelations, and (ii) conceptually, in domain models, detailing the application domain.

Automation assessment. As this activity is predominantly based on actual problem analysis, it is evident that it is mainly performed by human requirements engineers, possibly with the aid of CASE tools (e.g. UML tools, use case templates) to ensure the consistency and conformity of the resulting requirement artifacts.

\section{Activity 2. Identify and Separate Crosscut- ting Concerns in Requirements}

\section{2a. Identification.}

Several approaches exist automating the identification of crosscutting concerns in structured or unstructured requirements $[8,2,9,15]$. As these tools are based on Natural Language Processing (NLP), they employ pre-defined lexicons, which relate keywords to well-known crosscutting concerns. To identify a crosscutting concern in requirements, statistical analysis techniques are employed.

However these approaches and tools are reported to work well [8], they still require human input. Firstly, a lexicon is in fact a machine-readable representation of the experience gained by human requirement engineers in that particular domain. It comprises the typical crosscutting concerns, and provides information on how to identify them. Therefore, providing this information can only be done correctly by an experienced requirements engineer with ample experience in the problem domain under investigation. Secondly, a human post-processing step to identify and remove false positives might be necessary for reasons of quality control.

\section{2b. Separation.}

As outlined in Section 2, this involves the separation of concerns in use cases by introducing aspect use cases and relating these to the base use cases by means of use case extension.

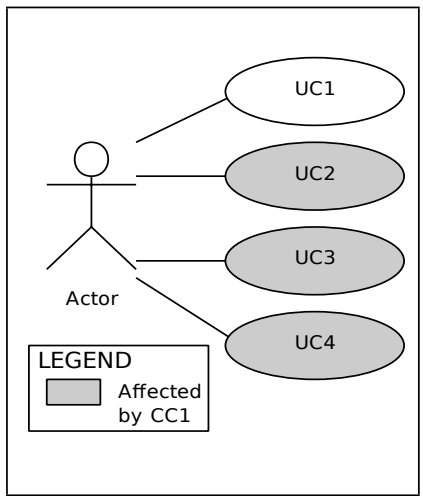

(a)

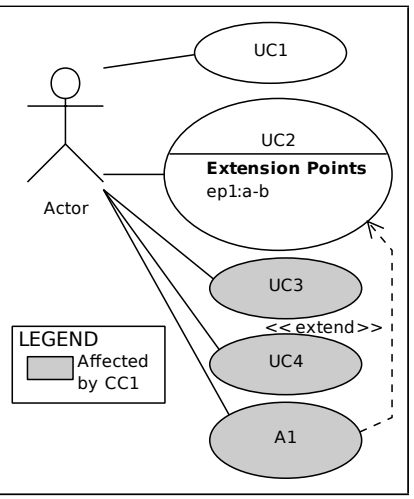

(b)
Figure 3: Illustration of the first two runs of the use case separation algorithm.

Algorithm. This activity is presented in pseudo-code below. The following functions and procedures are used:

- find_concern_steps (usecase, concern): This function returns for the use case 'usecase' the exact steps that are affected by the crosscutting concern 'concern'. This involves searching the result of activity $2 \mathrm{a}$, the identification of crosscutting concerns.

- find_aspect_uc(steps): This function searches the set of use cases for an aspect use case that offers the use case steps 'steps'. If no such use case exists, 'NULL' is returned.

- create_aspect_use_case(steps): This function creates a use case consisting of the use case steps 'steps' and returns it.

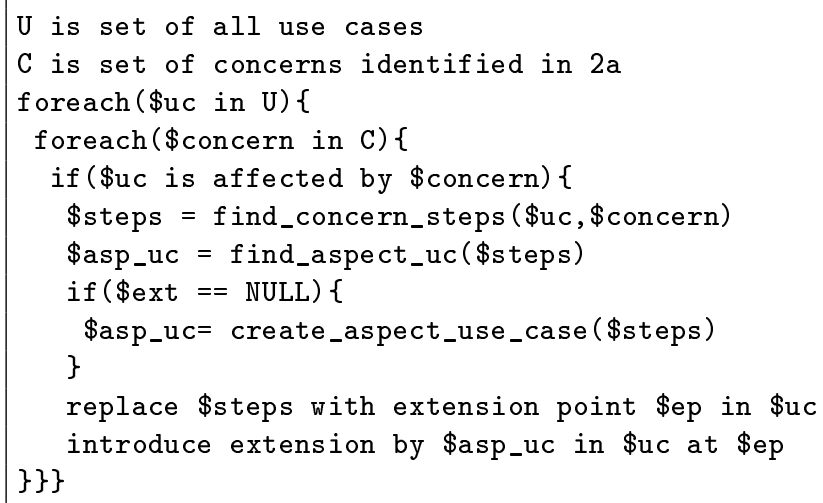

Illustration. Consider the use case diagram presented in Figure 3a. The actor is offered four distinct use cases: $\mathrm{UC} 1, \mathrm{UC} 2, \mathrm{UC} 3$, and UC4. In activity $2 \mathrm{a}$, one crosscutting concern was identified, $\mathrm{CC} 1$, which affects UC2, UC3 and UC4. 


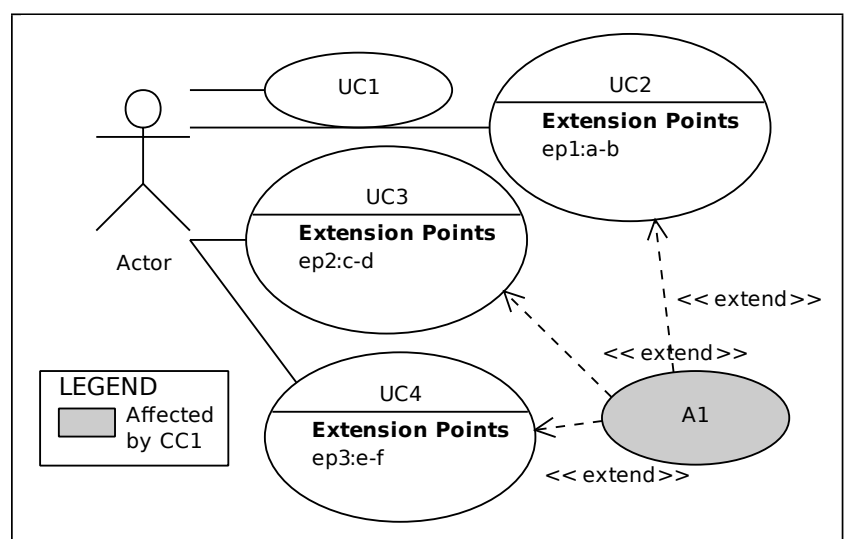

Figure 4: The final result of applying the use case separation algorithm on the diagram in Figure $3 \mathrm{a}$.

In the first run of the main loop (line 3) \$uc is UC1, which is not affected by CC1 (test at line 5).

In the second run of the loop (line 3), \$uc is UC2, which is affected by the crosscutting concern $\mathrm{CC} 1$ (test at line 5). Therefore, the affected steps are identified by the 'find_concern_steps' function (line 6). In this example, \$steps is $\{a, b\}$. Next, the set of use cases is searched for an aspect use case that offers these steps (the function 'find_aspect_uc' at line 7), but none is found (test at line $8)$. Therefore, a new use case A1 is created with steps $\{a-b\}$ (function 'create_aspect_use_case' at line 9). Finally, in $\mathrm{UC} 2$, the steps $\{\mathrm{a}-\mathrm{b}\}$ are replaced with an extension point named ep1 (line 11), and an extension relationship is introduced between UC2 and A1 (line 12). Figure 3b presents the result at this point.

In the third run of the main loop (line 3), \$uc is UC3. Again, this use case is affected by CC1 (test at line 5). And again, the affected steps are identified (line 6), in this case $\$$ steps is $\{\mathrm{c}-\mathrm{d}\}$. Next, to avoid duplicate aspect use cases, the set of use cases is searched for an aspect use case that offers these steps, and this time the use case A1 (defined in the second run of the main loop) is found (test at line 8). Finally, steps $\{\mathrm{c}, \mathrm{d}\}$ are replaced with an extension point named ep2 (line 11), and an extension relationship is introduced between UC3 and A1 (line 12).

The fourth and final run of the main loop is analogous to the third run, with $\$$ uc bound to UC4. The result after this run is presented in Figure 4.

Automation assessment. As illustrated above, this activity consists mainly of diagram transformations which are automatable. None of the presented steps or functions used in the algorithm essentially require human input.

\section{Activity 3. Generalize Crosscutting Use Case Extension Relationships}

Algorithm. Below, the generalization activity is presented algorithmically. The functions and procedures used in this algorithm are defined as follows:

- commonality (uc1, uc2, asp_uc): This function identifies a commonality between 'uc1' and 'uc2', more specifically at their respective extension point at which 'asp_uc' extends them. It returns a boolean value. The main question driving this is: is there an essential commonality at the extension points of 'uc1' and 'uc2', that they are both extended by 'asp_uc'?

- find_abstr_uc(uc1,uc2, asp_uc): This function searches the set of existing use cases for an abstract use case that can generalize the 'uc1' and 'uc2' use cases, and more specifically at their respective extension point at which 'asp_uc' extends them. If such abstract use case is found, this function returns it; otherwise 'NULL' is returned.

- generalize(uc1,uc2, asp_uc): this procedure creates an abstract use case that generalizes 'uc1' and 'uc2', more specifically at their respective extension point at which 'asp_uc' extends them. The commonalities at both extension points are generalized and moved higher up in the inheritance hierarchy. An important side-effect is that the concrete extension points are generalized as well.

- specialize(uc1,uc2, parent): this procedure introduces a specialization relation between 'uc1' and 'parent', and between 'uc2' and 'parent'.

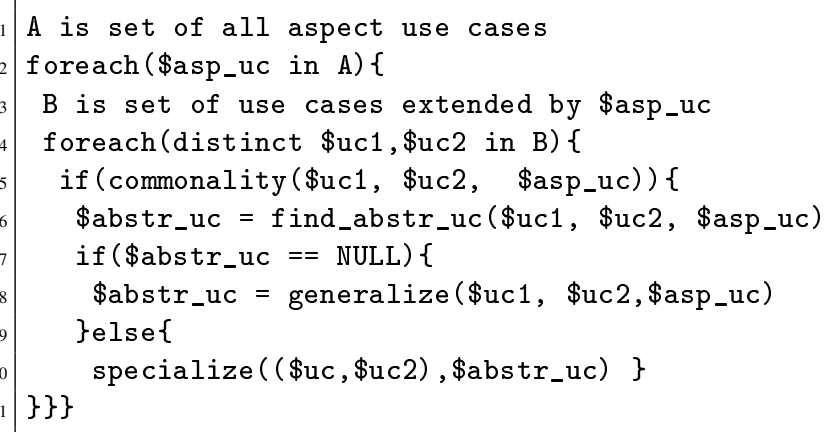

Illustration. We illustrate the generalization activity in the example of Figure 4. The initial relationships between the base use cases are summarized as follows: commonality (UC2, UC3, A1) is TRUE, commonality (UC2, UC4, A1) is FALSE, and therefore commonality (UC3, UC4, A1) is FALSE.

The main loop (line 2) will execute only once, as A contains only one aspect use case, and '\$asp_uc' is A1. The set of use cases extended by $\mathrm{A} 1$ (line 3 ) is $\mathrm{B}=$ $\{\mathrm{UC2}, \mathrm{UC} 3, \mathrm{UC} 4\}$. The first use case couple under consideration in the inner loop (line 4 ) is (UC2, UC3). As spec- 


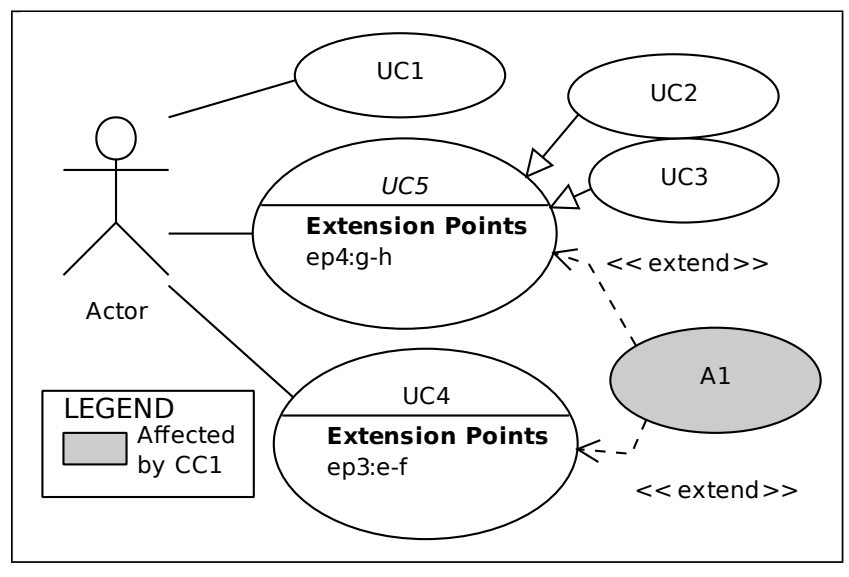

Figure 5: The final result after applying the generalization algorithm to the use cases of Figure 4.

ified above, a commonality exists between these use cases (test at line 5). The function 'find_abstr_uc' looks for an existing abstract use case that offers this commonality (line 6 ), but finds no such use case (test at line 7). Therefore, the 'generalize' function generalizes UC2 and UC3 (line 8), which leads to the introduction of the abstract use case UC5. As discussed above, the two concrete extension points, ep1 and ep2, are generalized as well. This leads to the introduction of the abstract extension point ep4 which points to the abstract steps $\{\mathrm{g}-\mathrm{h}\}$. The specialization relationship is structured in such a way that steps $\{a-b\}$ in UC2 and steps $\{\mathrm{c}-\mathrm{d}\}$ in UC3 specialize the steps $\{\mathrm{g}-\mathrm{h}\}$ of the abstract use case UC5.

The second use case couple under consideration in the inner loop (line 4) is (UC2, UC4). As stated above there is no commonality (test at line 8). The same applies to the third use case couple, (UC3, UC4). Finally the algorithm quits. The resulting diagram is presented in Figure 5.

Automation assessment. The 'commonality' and 'find_abstr_uc' functions and the 'generalize' procedure all involve analysing the semantics of the use cases under investigation, and identifying whether or not they have an essential commonality at their extension points. The 'generalize' procedure involves the generalizing concrete extension points. Selecting a name for a generalized extension point involves paying ample attention to finding the domain abstraction that best describes the semantics of the extension point in question. The guiding principle is that the domain abstractions should be anchored in the domain model. For these complex domain and requirements analysis activities, a human requirements or domain engineer is required.

\section{Activity 4. Define Discovered Domain Ab- stractions}

In the previous activity, extension points are introduced high up in the generalization hierarchy, and aspect use cases are composed to these. This activity involves a one-to-one mapping from these abstract extension points to domain abstractions. Additionaly, by explicitly specifying data flow requirements for each extension point, contextual information is defined. Indeed, it is often the case that the aspect use case requires information available only in the base use case, and needs to be passed on through the extension point ${ }^{2}$. The resulting domain abstraction comprises the name of the extension point and this contextual information.

This mapping is presented algoritmically below. The functions employed are:

- find_base_use_cases(ep): This function is used to search all use cases that offer the extension point 'ep', either explicitly, or by inheritance.

- find_aspect_use_cases(ep): This function returns the set of aspect use cases that have an extension relationship to extension point 'ep'.

- derive_data_flow (ep,B,A): This function returns the data flow requirements at the extension point 'ep'. This is done by analysing which information is required by the aspect use cases in 'A' and how it can be provided by the base use cases in ' $B$ '.

- create_domain_abstr(ep,dataflow): This function creates a new domain abstraction, based on the extension point 'ep' and data flow requirements 'dataflow'.

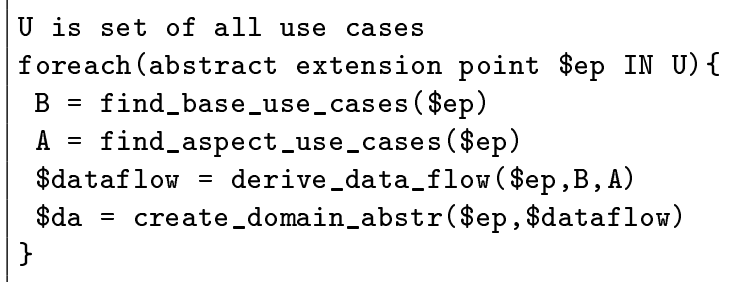

Automation assessment. As discussed above, the activities represented by the function 'derive_data_flow' require analysis of data flow between aspect and base use cases. This is a human activity, as it involves interpreting and analysing concrete use case steps. Other activities in this algorithm involve straightforward mappings, and are therefore automatable.

\footnotetext{
${ }^{2}$ Some aspect-oriented use case approaches such as AspectU [10] offer a mechanism to specify this information in the use case models themselves. However, to conform more closely to widely-applied use case modelling techniques, we specify data flow requirements at this point in our approach.
} 


\section{Activities 5 to 7 . Creating pointcut inter- faces in AO Architecture}

These activities involve (i) creating the architecture and constraining it for poincut interfaces (activity 5), (ii) implementing these pointcut interfaces in $\mathrm{AO}$ architecture (activity 6), and finally, (iii) creating $\mathrm{AO}$ compositions to the pointcut interfaces (activity 7 ). As already indicated in earlier work [14], this mostly involves mapping the domain abstractions to pointcut interfaces, and explicitizing the contextual information as pointcut parameters.

Automation assessment. Depending on many external factors such as the concrete architecture creation method used, and how well traceability information is kept during the creation of the architecture, assessing to which degree these activities can be automated is a very complex matter. Due to space constraints, we do not elaborate on this further.

\section{Related Work}

Synthesis Based Software Architecture Design. Tekinerdogan et al. [13] were among the first to identify the importance of domain analysis for deriving stable abstractions for aspects. In particular, their Synthesis Based Software Architecture method [12] proposes domain analysis techniques for building canonical models that identify the stable abstractions in a particular domain. This method however focuses mostly on deriving solution domain abstractions for aspects. In opposition, our work takes the approach of deriving problem domain abstractions for aspects. Clearly both views are necessary and complementary to each other. Therefore, exploring the integration of these two approaches is considered future work.

Early Aspects. AORE tool support from Lancaster University [8] already provides automated support for the identification of crosscutting concerns in requirements documentations. Moreover, their semantics-based composition approach to specify crosscutting dependencies between requirements in the RDL language [2] provides a solid basis for the dealing with fragility issues across the software development life-cycle. The COMPASS approach [1] illustrates this view by mapping these requirements-level aspect composition to architecture-level compositions at the architecture level. For this, COMPASS uses a one-to-one mapping between requirements-level and architecture-level compositions. In contrast, our method takes a many-to-one mapping approach, where a single pointcut signature at the architectural level is derived by generalizing multiple aspectual compositions at the requirements level. The generalization step in our approach is the key element to enables a higher reusability of pointcuts across multiple aspects.
AO Architecture. Kulesza, et al. [6] have identified a synergy between extension points in use cases and aspectual interfaces, for the purpose of improving the traceability between requirements and architecture. In opposition, our approach exploits this synergy to discover stable abstractions for reusable aspectual interfaces.

\section{Conclusion}

In this paper, we have assessed the feasibility of automating our method to discover stable domain abstractions in requirements for the purpose of defining reusable pointcut interfaces in AO architecture [14]. For each of the activities of this approach, we have presented an algoritmic view and discussed to which degree it can be automated. In summary: however most activities are well-suited for automation, the core of the approach -introducing abstract extension points in the base use case hierarchy - is by definition a human activity, as it involves interpreting the semantics of the requirements and refining the analysis of the problem domain.

\section{References}

[1] R. Chitchyan, M. Pinto, A. Rashid, and L. Fuentes. Compass: Composition-centric mapping of aspectual requirements to architecture. Transactions on Aspect-Oriented Software Development IV, pages 3-53, 2007.

[2] R. Chitchyan, A. Rashid, P. Rayson, and R. Waters. Semantics-based composition for aspect-oriented requirements engineering. In B. M. Barry and O. de Moor, editors, AOSD, volume 208 of ACM International Conference Proceeding Series, pages 36-48. ACM, 2007.

[3] E. Evans. Domain-Driven Design: Tackling Complexity in the Heart of Software. Addison-Wesley, 2004.

[4] I. Jacobson. Object-Oriented Software Engineering: A Use Case Driven Approach. Addison Wesley Longman Publishing Co., Inc., Redwood City, CA, USA, 2004.

[5] A. Kellens, K. Mens, J. Brichau, and K. Gybels. Managing the evolution of aspect-oriented software with modelbased pointcuts. In D. Thomas, editor, ECOOP, volume 4067 of Lecture Notes in Computer Science, pages 501-525. Springer, 2006.

[6] U. Kulesza, E. Alves, R. Garcia, A. C. Neto, E. Cirilo, C. J. P. D. Lucena, and P. Borba. Mapping features to aspects: A model-based generative approach. In Early Aspects: Current Challenges and Future Directions, volume 4765 of LNCS. Springer, 2007.

[7] J. Meekel, T. B. Horton, R. B. France, C. Mellone, and S. Dalvi. From domain models to architecture frameworks. In SSR '97: Proceedings of the 1997 symposium on Software reusability, pages 75-80, New York, NY, USA, 1997. ACM.

[8] A. Sampaio, A. Rashid, R. Chitchyan, and P. Rayson. Eaminer: Towards automation in aspect-oriented requirements engineering. In Transactions on Aspect-Oriented Software 
Development, III, pages 4-39. 2007. ISSN 0302-9743 (Print). 1611-3349 (Online).

[9] A. Sampaio, A. Rashid, and P. Rayson. Early-aim: An approach for identifying aspects in requirements. In $R E$ '05: Proceedings of the 13th IEEE International Conference on Requirements Engineering, pages 487-488, Washington, DC, USA, 2005. IEEE Computer Society.

[10] J. Sillito, C. Dutchyn, A. D. Eisenberg, and K. D. Volder. Use case level pointcuts. In M. Odersky, editor, ECOOP, volume 3086 of Lecture Notes in Computer Science, pages 244-266. Springer, 2004.

[11] I. Sommerville. Software Engineering. Pearson, 2008.

[12] B. Tekinerdogan and M. Aksit. Synthesis based software architecture design. In Software Architectures and Component Technology.

[13] B. Tekinerdogan and M. Aksit. Deriving design aspects from conceptual models. In ECOOP '98: Workshop on ObjectOriented Technology, pages 410-413, London, UK, 1998. Springer-Verlag.

[14] D. Van Landuyt, S. Op de beeck, E. Truyen, and W. Joosen. Domain-driven discovery of stable abstractions for pointcut interfaces. In AOSD '09: Proceedings of the 8th international conference on Aspect-oriented software development, pages 1-10, New York, NY, USA, 2009. ACM.

[15] Y. Yu, J. C. S. do Prado Leite, and J. Mylopoulos. From goals to aspects: Discovering aspects from requirements goal models. In $R E$, pages 38-47. IEEE Computer Society, 2004. 